\title{
Methamphetamine use association with pulmonary diseases: a retrospective investigation of hospital discharges in California from 2005 to 2011
}

\author{
Halley Tsai ${ }^{1}$, Justin Lee ${ }^{2}$, Haley Hedlin $\mathbb{0}^{2}$, Roham T. Zamanian ${ }^{1,3,4}$ and \\ Vinicio A. de Jesus Perez ${ }^{1,3,4}$
}

Affiliations: ${ }^{1}$ Division of Pulmonary and Critical Care Medicine, Stanford University, Stanford, CA, USA ${ }^{2}$ Quantitative Sciences Unit, Stanford University, Stanford, CA, USA. ${ }^{3}$ Vera Moulton Wall Center for Pulmonary Vascular Disease, Stanford University, Stanford, CA, USA. ${ }^{4}$ Both authors contributed equally.

Correspondence: Vinicio A. de Jesus Perez, Division of Pulmonary and Critical Care Medicine, Stanford University Medical Center, 300 Pasteur Drive, Grant S140b, Stanford, CA 94305, USA. E-mail: vdejesusa stanford.edu

\section{ABSTRACT}

Background: Methamphetamine can have acute and long-term adverse health consequences. Our objective was to determine whether methamphetamine use is associated with more hospitalisation codes for asthma exacerbation, chronic obstructive pulmonary disease (COPD) exacerbation, pneumonia and acute respiratory failure $(\mathrm{ARF})$.

Methods: The Health Care Utilization Project (HCUP) database includes retrospective inpatient discharge abstracts from 2005 through 2011 from the California state inpatient databases (SIDs). ICD-9 codes were used to identify hospitalisations for asthma exacerbation, COPD exacerbation, acute pneumonia, ARF and methamphetamine use from discharges with complete demographic data and ages 18 to 75 years. Adjusted rate ratios comparing methamphetamine users with nonusers were estimated separately for each pulmonary disease diagnosis by sex using negative binomial regression models.

Results: We included 21125249 inpatient discharges from 2005 through 2011 in California in our analysis; $182766(0.87 \%)$ had methamphetamine use. The rate ratio comparing pneumonia in discharges with methamphetamine use versus those without were 1.40 (95\% CI 1.18, 1.67) for women and 1.18 (95\% CI 1.04, 1.35) for men; comparing ARF 1.77 (95\% CI 1.59, 1.98) for women and 1.24 (95\% CI 1.12, 1.37) for men; and comparing COPD exacerbation 1.40 (95\% CI 1.18, 1.67) for women and 0.90 (95\% CI 0.79, 1.02) for men.

Conclusions: A positive association was found when comparing inpatient hospital discharge diagnoses for methamphetamine use and those for pneumonia and ARF in both sexes. This association was not seen when comparing discharge diagnoses for methamphetamine and those for asthma exacerbation in both sexes or COPD exacerbation in men. While future investigation for is warranted, this finding may help to further characterise the pulmonary toxicity of methamphetamine.

@ERSpublications

A positive association was found when comparing inpatient hospital discharge diagnoses for methamphetamine use and those for pneumonia and respiratory failure in both sexes http://bit. ly/2Jem $87 \mathrm{z}$

Cite this article as: Tsai $\mathrm{H}$, Lee $\mathrm{J}$, Hedlin $\mathrm{H}$, et al. Methamphetamine use association with pulmonary diseases: a retrospective investigation of hospital discharges in California from 2005 to 2011. ERJ Open Res 2019; 5: 00017-2019 [https://doi.org/10.1183/23120541.00017-2019].

This article has supplementary material available from openres.ersjournals.com.

Access to the HCUP database must be requested through the HCUP Central distributor: https://www.hcup-us.ahrq.gov/ tech_assist/centdist.jsp.

Received: 15 Jan 2019 | Accepted after revision: 01 July 2019

Copyright $\odot E R S$ 2019. This article is open access and distributed under the terms of the Creative Commons Attribution Non-Commercial Licence 4.0. 


\section{Introduction}

The methamphetamine epidemic in the United States has been widely characterised as a significant public health concern. Methamphetamine is a psychostimulant of the phenethylamine and amphetamine classes of drugs and is a sympathomimetic that accentuates catecholaminergic and serotonergic neurons [1]. Its precursor forms were initially used to treat asthma and sinus congestion (ephedrine) [2], and as a weight loss aid (fen-phen) [3]. The discovery of its stimulant effects in the early 20th century led to its widespread use as a performance-enhancer, then subsequently as a recreational drug of abuse in purified forms. Specifically, methamphetamine is sold illicitly as a powder or crystalline ("ice" or "crystal") form, intended for inhalation via smoking, the most common route of ingestion [1].

According to recent estimates [4] from 2012 in the United States, over 12 million people over the age of 12 years have used methamphetamine in their lifetimes, 1.2 million people reported having used in that past year, and 440000 used it in that past month. According to the National Institute on Drug Abuse 2012 data, methamphetamine use was greatest in the western part of the United States and parts of the Midwest, and users were predominately White [5]. Outside of the United States, amphetamine-type stimulants, of which methamphetamine is the most common, are the second-most used class of illicit drugs worldwide [6]. Furthermore, the World Drug Report of 2013 estimates that methamphetamine use appears to be growing, further suggesting its use as a growing epidemic [7].

Because of the increased rates of methamphetamine use, there has been subsequent investigation into its toxic effects on health. In 2010, Volkow et al. [8] showed that the highest uptake of methamphetamine was in the lungs, liver, and kidney, suggesting its widespread organ distribution and potential for toxicity. Previously reported associations have been predominantly in the cardiac and respiratory systems, namely cardiomyopathy, acute coronary syndrome, and aortic dissection, as well as pulmonary arterial hypertension [9-11]. Small studies also exist to suggest the possible associations between methamphetamine use and barotrauma, cardiogenic pulmonary oedema, pulmonary haemorrhage, pulmonary granulomatosis, noncardiogenic pulmonary oedema, aspiration pneumonia, excessive bronchial reactivity, and hypersensitivity pneumonitis [12]. However, there is a paucity of investigation towards the scope of pulmonary toxicity that may be related to methamphetamine use.

Thus, the goal of this study was to determine whether methamphetamine use was associated with more hospitalisation codes for adults which capture four common pulmonary diseases: acute exacerbations of asthma, acute exacerbations of chronic obstructive pulmonary disease (COPD), pneumonia and acute respiratory failure. Our target of study focused on the data collected from California, a state that has been the focus of methamphetamine-related research in the past [13]

\section{Materials and methods}

\section{Study design and population}

A retrospective observational study design was used to analyse California state inpatient databases (SIDs) obtained from the Healthcare Cost and Utilization Project (HCUP; www.hcup-us.ahrq.gov/tech_assist/ citations.jsp) from 2005 through 2011 for diagnosis of methamphetamine use and asthma exacerbation, COPD exacerbation, pneumonia and respiratory failure. Inpatient hospital discharges were included in the study population if patients were over 18 years old and were not missing data for key demographic variables (age, sex, estimated median household income of residents in the patient's ZIP code).

\section{Outcomes}

Primary outcomes were discharge diagnoses of four common pulmonary diseases: acute exacerbations of asthma, COPD, pneumonia and acute respiratory failure. The relevant diagnoses were defined by International Classification of Diseases, Ninth Revision (ICD-9) codes documented in the top 10 diagnoses for each discharge (table 1). Specific ICD-9 codes were chosen to capture discharge diagnoses of acute exacerbations of asthma and $\mathrm{COPD}$, respiratory failure and diagnoses consistent with community-acquired pneumonia. Discharges with more than one of the diagnoses of interest were classified as having each of the diagnoses.

\section{Independent variable and covariates}

The exposure of interest was methamphetamine use (ICD-9 codes 304.40 to 304.42 and 305.70 to 305.72) in the top 10 diagnoses for each discharge. The selected methamphetamine use diagnoses included unspecified, continuous or episodic abuse or dependence. ICD-9 codes for patients in remission of methamphetamine abuse were excluded. In addition, we identified tobacco use (ICD-9 codes 305.1, 989.84, V15.82) as a possible confounder. The use of ICD-9 tobacco use codes for identifying smokers in a clinical population, while insensitive, has been supported in the literature [14]. Age, sex, race and median household income were used as demographic covariates. Age (18 to 25,26 to 35,36 to 45,46 to 55,56 to 


\begin{tabular}{|c|c|c|}
\hline Diagnosis & $\begin{array}{l}\text { Diagnosis } \\
\text { code }\end{array}$ & Description \\
\hline \multirow[t]{6}{*}{ Methamphetamine } & 304.40 & Amphetamine and other psychostimulant dependence, unspecified \\
\hline & 304.41 & Amphetamine and other psychostimulant dependence, continuous \\
\hline & 304.42 & Amphetamine and other psychostimulant dependence, episodic \\
\hline & 305.70 & $\begin{array}{c}\text { Amphetamine or related acting sympathomimetic abuse, } \\
\text { unspecified }\end{array}$ \\
\hline & 305.71 & $\begin{array}{c}\text { Amphetamine or related acting sympathomimetic abuse, } \\
\text { continuous }\end{array}$ \\
\hline & 305.72 & Amphetamine or related acting sympathomimetic abuse, episodic \\
\hline \multirow[t]{6}{*}{ Asthma } & 493.01 & Extrinsic asthma with status asthmaticus \\
\hline & 493.02 & Extrinsic asthma with (acute) exacerbation \\
\hline & 493.11 & Intrinsic asthma with status asthmaticus \\
\hline & 493.12 & Intrinsic asthma with (acute) exacerbation \\
\hline & 493.91 & Asthma, unspecified type, with status asthmaticus \\
\hline & 493.92 & Asthma, unspecified type, with (acute) exacerbation \\
\hline \multirow[t]{2}{*}{ COPD } & 491.21 & Obstructive chronic bronchitis with (acute) exacerbation \\
\hline & 491.22 & Obstructive chronic bronchitis with acute bronchitis \\
\hline \multirow[t]{12}{*}{ Pneumonia } & 481.0 & Pneumococcal pneumonia (Streptococcus pneumoniae pneumonia) \\
\hline & 482.2 & Pneumonia due to Haemophilus influenzae \\
\hline & 482.30 & Pneumonia due to Streptococcus, unspecified \\
\hline & 482.31 & Pneumonia due to Streptococcus, group A \\
\hline & 482.32 & Pneumonia due to Streptococcus, group B \\
\hline & 482.39 & Pneumonia due to other Streptococcus \\
\hline & 482.40 & Pneumonia due to Staphylococcus, unspecified \\
\hline & 482.41 & Methicillin susceptible pneumonia due to Staphylococcus aureus \\
\hline & 482.42 & Methicillin resistant pneumonia due to Staphylococcus aureus \\
\hline & 482.49 & Other Staphylococcus pneumonia \\
\hline & 482.84 & Pneumonia due to Legionnaires' disease \\
\hline & 483.0 & Pneumonia due to Mycoplasma pneumoniae \\
\hline Respiratory failure & 518.81 & Acute respiratory failure \\
\hline \multirow[t]{3}{*}{ Tobacco } & 305.1 & Tobacco use disorder \\
\hline & 989.84 & Toxic effect of tobacco \\
\hline & V15.82 & Personal history of tobacco use \\
\hline \multirow[t]{6}{*}{ Cocaine } & 304.20 & Cocaine dependence, unspecified \\
\hline & 304.21 & Cocaine dependence, continuous \\
\hline & 304.22 & Cocaine dependence, episodic \\
\hline & 305.60 & Cocaine abuse, unspecified \\
\hline & 305.61 & Cocaine abuse, continuous \\
\hline & 305.62 & Cocaine abuse, episodic \\
\hline
\end{tabular}

65, 66 to 75 years) and race (White, Hispanic, other/missing) were grouped together for the analysis while sex and median household income quartiles [1-4] were used as documented in the database. On the basis of published demographic data, age ranges were centred above and below age 35 years, the median age of methamphetamine abusers, with a standard deviation of approximately 6 years and range from approximately age 20 to 70 years $[13,15]$.

Cocaine is also a sympathomimetic drug whose abuse has been documented to provoke and/or exacerbate the four lung diseases presented here $[12,16-20]$. To serve as a comparison with a well-studied toxin, cocaine abuse and dependence (ICD-9 codes 304.20 to 304.22 and 305.60 to 305.62) in the top 10 diagnoses was considered as the exposure in sensitivity analyses.

\section{Statistical analysis}

Rates of discharge diagnoses are presented as the number of discharges having that diagnosis in the numerator and the total number of discharges in the denominator.

Negative binomial regression models were used to estimate rate ratios (RR) with 95\% confidence intervals (CIs) of pulmonary disease diagnoses between methamphetamine and nonmethamphetamine users. Models were adjusted for age group, race, median household income quartile and smoking. Given the 
known disparity in disease risk by sex [21-23], we fitted separate models for each pulmonary disease diagnosis by sex to estimate the RR of the disease in methamphetamine versus nonmethamphetamine users separately by sex. The same methods were applied in an analysis considering cocaine use as the exposure to provide a comparison with a well-studied toxin.

All statistical tests were performed at the 0.05 significance level and R [24] (version 3.3.1) was used for all analyses. The "MASS" [25] package (version 7.3-47) in R was used to fit the negative binomial regression models.

\section{Results}

Screening the California HCUP SID between 2005-2011 resulted in a total of 27907535 discharge abstracts that were available for analysis. Abstracts that reflected children (age <18 years, $\mathrm{n}=5594831$ ) or those with missing age $(n=266198)$ were excluded from further analysis. For the remaining abstracts, we excluded those with either missing sex $(n=341540)$, income $(n=563735)$ or both $(n=15982)$. This resulted in 21125249 discharge abstracts with complete demographic data for inclusion in our study, of which 182 766 carried a top-10 discharge diagnosis of methamphetamine abuse or dependence based on our inclusion criteria (figure 1).

With regard to ethics approval and consent to participate, all the data used in the study were obtained from the HCUP database.

\section{Demographics}

Table 2 summarises demographic data of the discharge diagnoses that were included in the study. The median age was 37 years (interquartile range (IQR) 28-47) in methamphetamine use discharges and 57 (IQR 37-75) for nonmethamphetamine use discharges. Methamphetamine use discharges tended to be male $(n=106665,58 \%)$ compared with nonuse $(n=7982930,38 \%)$. A co-diagnosis of methamphetamine and tobacco use $(n=68312,37 \%)$ occurred more frequently compared with diagnoses of tobacco use without methamphetamine use diagnosis $(n=3028832,14 \%)$. Rates of discharge diagnoses for both methamphetamine and nonmethamphetamine use in smokers and nonsmokers are presented by age and sex in supplementary figure 1 and 2 .

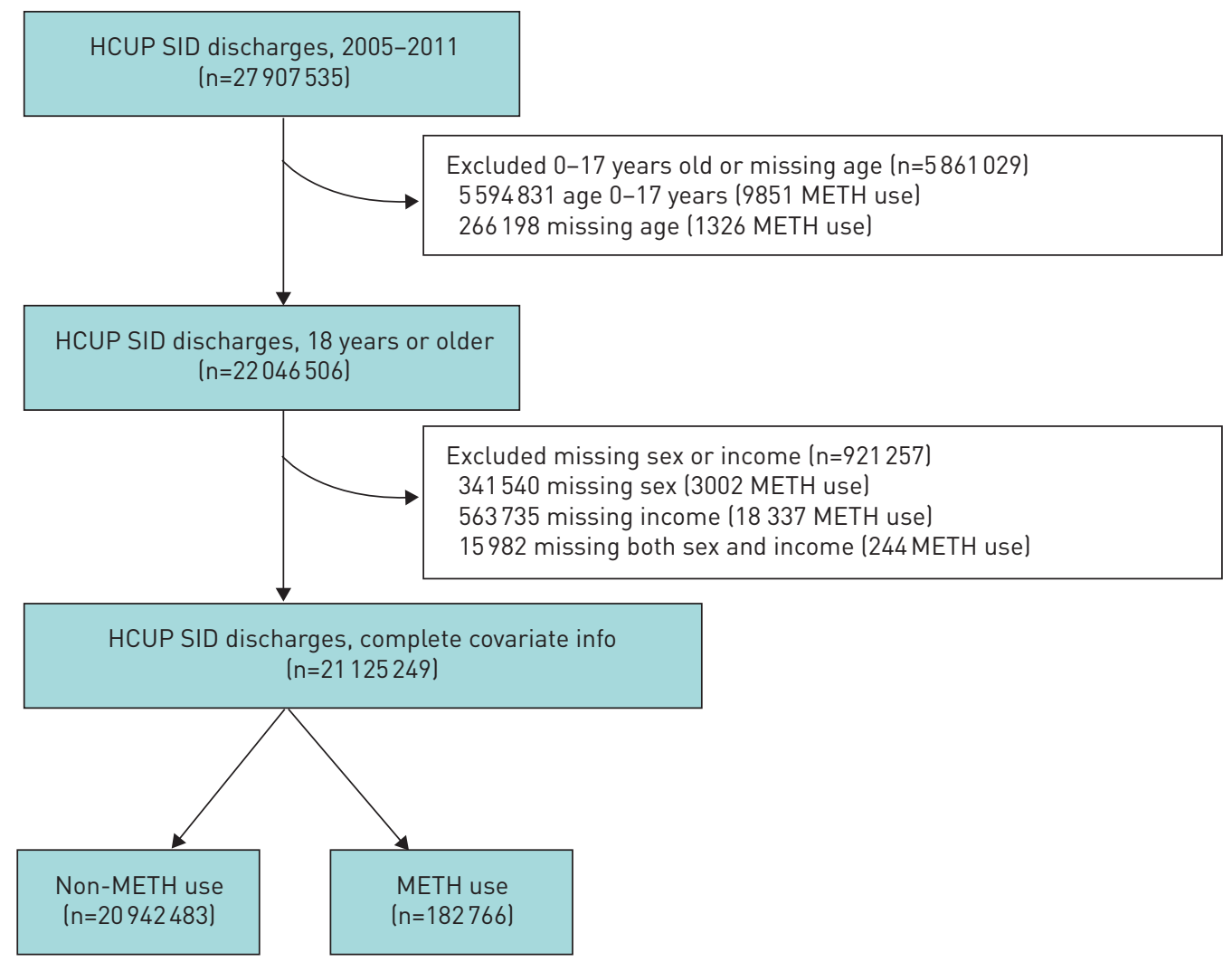

FIGURE 1 Number of discharges included and excluded in analysis. HCUP: Healthcare Utilization Project; SID: state inpatient database; METH: methamphetamine. 


\section{TABLE 2 California state inpatient database characteristics 2005-2011}

\begin{tabular}{|c|c|c|c|}
\hline & $\begin{array}{l}\text { Meth discharges ( } \mathrm{n}=182 \\
766)\end{array}$ & $\begin{array}{l}\text { Nonmeth discharges } \\
(n=20942483)\end{array}$ & SMD \\
\hline Age group & & & 1.229 \\
\hline $18-25$ years & 32151 (18\%) & $1862559(8 \%)$ & \\
\hline $26-35$ years & $48357(26 \%)$ & $3008855(14 \%)$ & \\
\hline $36-45$ years & $51283(28 \%)$ & $2329449(11 \%)$ & \\
\hline $46-55$ years & $38918(21 \%)$ & $2790528(13 \%)$ & \\
\hline $56-65$ years & $10540(6 \%)$ & $2991208(14 \%)$ & \\
\hline $66-75$ years & $1517(1 \%)$ & $7959884(36 \%)$ & \\
\hline Age years median (IQR) & $37(28,47)$ & $57(37,75)$ & 1.065 \\
\hline Sex & & & 0.414 \\
\hline Male & 106665 (58\%) & 7982930 (36\%) & \\
\hline Female & $76101(42 \%)$ & $12959553(59 \%)$ & \\
\hline Race & & & 0.127 \\
\hline White & $104846(57 \%)$ & $11150306(53 \%)$ & \\
\hline Hispanic & $46532(26 \%)$ & $5186339(25 \%)$ & \\
\hline Black & $12550(7 \%)$ & $1679988(8 \%)$ & \\
\hline Other/missing & $18838(10 \%)$ & $2925850(14 \%)$ & \\
\hline $\begin{array}{l}\text { Median household income quartile by } \\
\text { patient ZIP code }\end{array}$ & & & 0.279 \\
\hline Quartile 1 & $65813(36 \%)$ & $5785651(26 \%)$ & \\
\hline Quartile 2 & $53387(29 \%)$ & $5312536(24 \%)$ & \\
\hline Quartile 3 & $39373(22 \%)$ & $5237059(24 \%)$ & \\
\hline Quartile 4 & $24193(13 \%)$ & $4607237(21 \%)$ & \\
\hline Smoking & $68312(37 \%)$ & $3028832(14 \%)$ & 0.542 \\
\hline \multicolumn{4}{|l|}{ Pulmonary disease diagnoses } \\
\hline Asthma & $1076(1 \%)$ & $106712(0.5 \%)$ & 0.011 \\
\hline COPD & $2083(1 \%)$ & $519148(2 \%)$ & 0.101 \\
\hline Pneumonia & $898(0.5 \%)$ & $99947(0.5 \%)$ & 0.002 \\
\hline Respiratory failure & $4031(2 \%)$ & $463840(2 \%)$ & 0.001 \\
\hline More than one of above & $690(0.4 \%)$ & $93378(0.4 \%)$ & 0.01 \\
\hline
\end{tabular}

\section{Outcomes}

Discharges abstracts that included ICD-9 codes for methamphetamine use were 0.66 (95\% CI $0.57,0.77$; $\mathrm{p}<0.001$ ) times as likely to have a simultaneous principal hospital discharge abstract for acute exacerbations of asthma compared with discharge abstracts without methamphetamine use in women and 0.71 (95\% CI 0.61, 0.82; p<0.001) times as likely in men (figure 2). For COPD, the risk of having a principal hospital discharge abstract for acute exacerbations were 1.23 (95\% CI 1.06, 1.42; $\mathrm{p}=0.01)$ times as likely in discharges with methamphetamine use versus nonmethamphetamine use in women and 0.90 (95\% CI 0.79, 1.02; p=0.10) in men. We documented a RR for having a hospital discharge abstract for acute pneumonia in methamphetamine use versus nonmethamphetamine use of 1.40 (95\% CI 1.18, 1.67; $\mathrm{p}<0.001)$ in women and 1.18 (95\% CI 1.04, 1.35; p=0.01) in men. Finally, the risk in methamphetamine use discharges of also having a discharge diagnosis of acute respiratory failure was 1.77 (95\% CI 1.59, 1.98; $\mathrm{p}<0.001)$ times the risk in nonmethamphetamine use discharges in women and 1.24 (95\% CI 1.12, 1.37; $\mathrm{p}<0.001)$ in men. Similar results were found when adjusted for a confounder of malnutrition, and results are presented in Supplementary Figure 3.

\section{Role of cocaine in discharge for lung diseases}

In our analysis, we found similarities between cocaine use and methamphetamine use discharges associated with each of the four lung diagnoses (figure 3). In particular, we found a RR for asthma exacerbations of 0.87 (95\% CI $0.75,1.02 ; \mathrm{p}=0.08)$ when comparing discharges with cocaine use versus discharges without cocaine use in men and 0.71 (95\% CI $0.60,0.83 ; \mathrm{p}<0.001)$ in women. Compared with discharges with methamphetamine use, the RR for COPD exacerbations in discharges with cocaine use was higher in women (RR: 2.46; $95 \%$ CI 2.11, 2.86; $\mathrm{p}<0.001$ ) and men (RR: $1.69 ; 95 \%$ CI 1.47, 1.94; $\mathrm{p}<0.001)$. For pneumonia, discharges with cocaine use were $1.84(95 \%$ CI 1.55, 2.20; $\mathrm{p}<0.001)$ times as likely to have a pneumonia diagnosis compared with discharges without use in women and 0.88 (95\% CI 


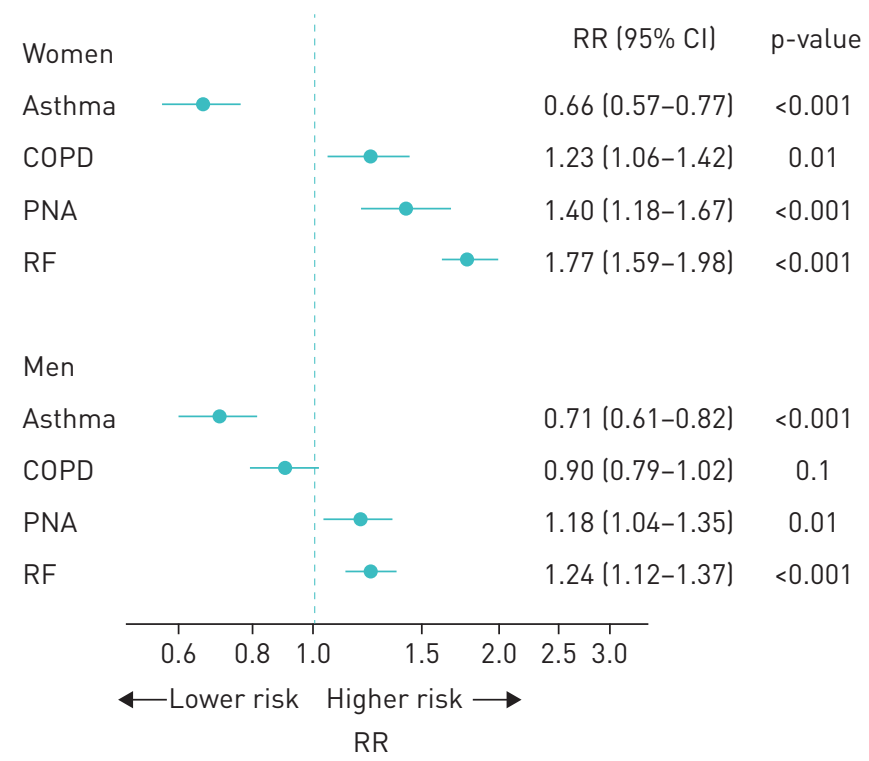

FIGURE 2 Rate ratio (RR) point estimates and 95\% confidence intervals for each disease in methamphetamine versus nonmethamphetamine discharges for females and males. Models adjusted for race, median household income, age and smoking status. COPD: chronic obstructive pulmonary disease; PNA: pneumonia; RF: respiratory failure.

$0.76,1.02 ; \mathrm{p}=0.08)$ in men; for acute respiratory failure, the RR was $1.73(95 \%$ CI $1.53,1.95 ; \mathrm{p}<0.001)$ in women and 1.05 (95\% CI 0.94, 1.16; $\mathrm{p}=0.39)$ in men.

\section{Discussion}

Our findings suggest that California inpatient hospitalisation discharge abstracts from the years 2005 through 2011 that include a code for methamphetamine use have an increased likelihood of also including a code for COPD exacerbation, acute pneumonia or acute respiratory failure when compared with discharge abstracts that do not include a code for methamphetamine use.

In our analysis, we found negative associations in both sexes between diagnosis codes for methamphetamine use and acute asthma exacerbation. This would suggest a decreased likelihood of

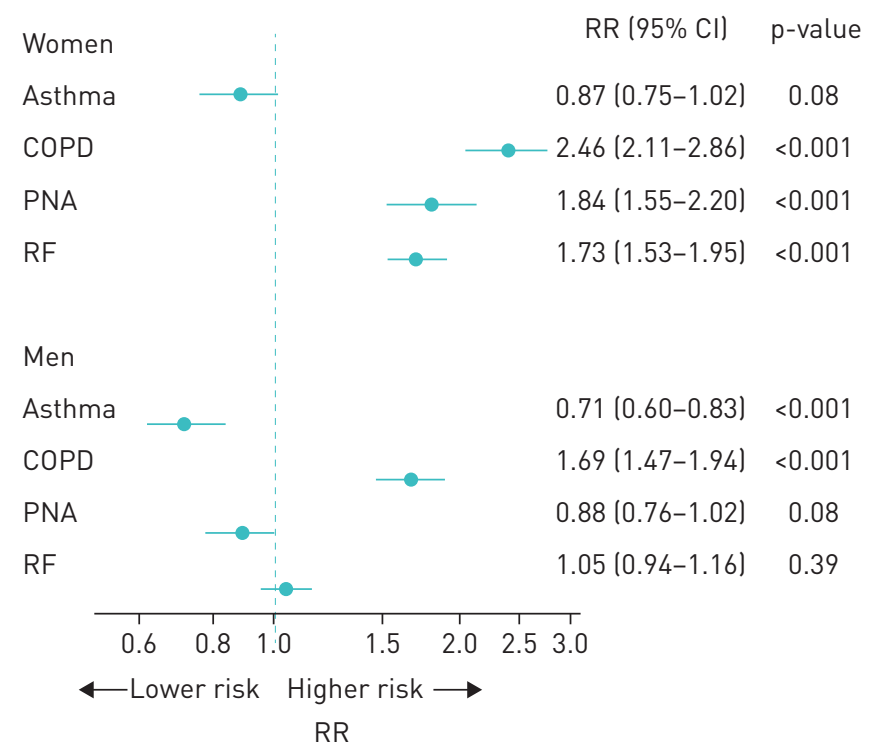

FIGURE 3 Rate ratio (RR) point estimates and 95\% confidence intervals (CI) for each disease in cocaine versus noncocaine discharges for females and males. Models adjusted for race, median household income, age, and smoking status. COPD: chronic obstructive pulmonary disease; PNA: pneumonia; RF: respiratory failure. 
concurrent discharge abstract diagnoses of methamphetamine use and acute asthma exacerbation that is contrary to our hypothesis that methamphetamine use may increase the risk of exacerbation. To our knowledge, there are no previously published data on the inhaled effects of methamphetamine with regards to airways diseases such as asthma. It is possible that this effect reflects a decreased likelihood for patients with concurrent discharge diagnoses to seek medical care, due to socioeconomic factors or mental health barriers that limit access to medical care. Another explanation for the RR being lower for asthma (and higher for COPD in women) may be that asthma is more commonly misdiagnosed as COPD in the presence of methamphetamine use. Finally, we acknowledge that the findings may reflect a limitation of the study design using ICD-9 codes as surrogates for exposure to methamphetamine and diagnosis of asthma exacerbation.

We identified a statistically significant positive association between diagnosis codes for methamphetamine use and acute COPD exacerbation in women. Surprisingly, there is scant literature regarding inhaled illicit drug use and rates of COPD exacerbation. While "crack" cocaine has been linked to increased risk of asthma exacerbations [26], most studies have failed to find inhaled marijuana to be a risk factor for development of COPD or increased rate of exacerbations [27]. To our knowledge, there are no published studies investigating a link between COPD and methamphetamine use. It is unclear why this positive association is seen in women but not is not statistically significant in men. In the original landmark TORCH COPD study, women did report more exacerbations and worse dyspnoea scores than men [22] thus, perhaps there was an increased propensity for women methamphetamine abusers to seek medical care for a concurrent COPD exacerbation.

The association between diagnosis codes for methamphetamine use and codes for the adverse pulmonary outcomes of COPD exacerbation, acute pneumonia or acute respiratory failure is in concordance with known pulmonary complications of acute respiratory failure related to another similar inhaled stimulant, "crack" cocaine [26]. Furthermore, there is some literature that describes a disruption by methamphetamine exposure on host immunity, placing methamphetamine users at increased risk for acquisition of diverse pathogens [28]. While much of the existing literature addresses increased rates of opportunistic infections and fungal infections such as histoplasmosis, methamphetamine has been reported to reduce $\mathrm{T}$-cell infiltrates in the lungs, dampening the protective immune response against respiratory pathogens including community-acquired bacteria [29]. Also, the intoxicating effects of methamphetamine predisposes users to engage in risky behaviour and subsequently increases the risk of acquiring transmissible microbes or developing immunodeficiency (e.g. HIV and AIDS) [28]. Another interpretation of this association is the possibility that patients who are afflicted by substance abuse may have poorer access to general outpatient medical care, and in turn present to the hospital more acutely and would require inpatient stay for their acute illness.

Lastly, as the specific ICD-9 code for "acute respiratory failure" is broad, it may encompass all forms of respiratory failure, including pulmonary oedema (cardiogenic or noncardiogenic), acute respiratory distress syndrome or diffuse alveolar damage, all entities which have been linked to methamphetamine use in case reports [30]. It has been described during investigation of the link between methamphetamine use and pulmonary hypertension that methamphetamine exposure potentiates DNA damage in hypoxic cells, increasing mitochondrial reactive oxygen species [31]. As acute lung injury and acute respiratory distress syndrome have been shown to arise from free radical formation [32], it is possible that methamphetamine use via free radical formation may contribute to noncardiogenic pulmonary oedema and capillary leak [33].

Limitations to this study include that this was a retrospective study that used data collected from a database originally created for billing purposes. Because the database was not designed for the purpose of research, there was limited information available on potential confounders and some confounder data were missing. It is possible that the missingness differs by methamphetamine exposure. Other than adjusting for obvious confounders that were available information from the database, including malnutrition (supplementary figure 3), there are probably other confounders that were not addressed (geographic location, domestic conditions, psychosocial environment, access to healthcare). This work presents several comparisons and the p-values should only be considered as descriptive measures that are not adjusted for the multiple tests performed. Additionally, by limiting our analysis to hospitalisation discharges, we cannot be certain whether the observed associations hold in the general population.

Furthermore, the use of ICD-9 codes as accurate surrogates for exposure (methamphetamine and tobacco) and disease state (asthma exacerbation, COPD exacerbation, pneumonia, respiratory failure) is dependent on the coders' accuracy in documenting the pertinent clinical problems for a given hospital discharge abstract. Misclassification is likely to attenuate the true RRs. Due to the nature of the dataset utilised, analysis was performed on discharge abstracts rather than on an individual basis because not all records could be linked to an individual. As a result, the temporality of the exposure is unknown in this study; for 
example, in the absence of urine toxicology data confirming methamphetamine exposure, a methamphetamine use ICD-9 code may still be documented based on history of use.

Future directions of investigation include validating this research question in the general population and further stratifying those with simultaneous diagnoses of methamphetamine use and community-acquired pneumonia by co-diagnosis of immunocompromised state such as HIV or AIDS based on proposed mechanisms of methamphetamine and infectious diseases. Many of the discharge abstracts available in the dataset are those of the oldest patients. Future research should evaluate whether the associations observed here vary by age. Furthermore, as recreational methamphetamine production has become purer over time [34], an analysis stratified by year of discharge may provide insight into changes in the relationship between methamphetamine use and lung disease over time.

\section{Conclusion}

When adjusted for age, race, economic quartile and tobacco smoking, discharge diagnoses with methamphetamine use diagnoses are associated with higher rates of concurrent diagnoses of community-acquired pneumonia and acute respiratory failure among men and women, and acute COPD exacerbation among women when compared with those with no methamphetamine use diagnosis. Further investigation is necessary to elucidate a potential mechanism on how methamphetamine use leads to development of these common pulmonary diagnoses. We hope that this study brings awareness to the growing methamphetamine epidemic and its potential for lung injury beyond the pulmonary vasculature and the cardiopulmonary system, namely the airways and lung parenchyma. Lastly, there is also potential for further investigation with regards to the healthcare cost of the methamphetamine epidemic and utilisation of services of the healthcare system.

Author contributions: H. Tsai, J. Lee, H. Hedlin, R.T. Zamanian and V.A. de Jesus Perez conceived and designed the study. H. Tsai and J. Lee analysed the datasets and performed the statistical analysis under the supervision of H. Hedlin, R.T. Zamanian and V.A. de Jesus Perez. All authors take responsibility for the integrity of the data presented in the manuscript.

Conflict of interest: None declared.

Support statement: This work was supported by US National Institutes of Health grants R01 HL134776, R01 HL139664 and R03 HL133423-01, an AHA Beginning Grant in Aid, and a Stanford Cardiovascular Institute and Translational Research and Applied Medicine grant to V.A. de Jesus Perez. Funding information for this article has been deposited with the Crossref Funder Registry.

\section{References}

1 Courtney KE, Ray LA. Methamphetamine: an update on epidemiology, pharmacology, clinical phenomenology, and treatment literature. Drug Alcohol Depend 2014; 143: 11-21.

2 Aronson JK. Ephedra, ephedrine, and pseudoephedrine. In: Aronson JK, ed. Meyler's Side Effects of Drugs. 16th Edn. Amsterdam, Elsevier B.V., 2016; pp. 65-76.

3 Ciccarone D. Stimulant abuse: pharmacology, cocaine, methamphetamine, treatment, attempts at pharmacotherapy. Prim Care 2011; 38: 41-58.

4 Substance Abuse and Mental Health Services Administration. Results from the 2012 National Survey on Drug Use and Health: Summary of National Findings, NSDUH Series H-46, HHS. Rockville, SAMHS, 2013.

5 National Institute on Drug Abuse. Epidemiologic Trends in Drug Abuse. Proceedings of the Community Epidemiology Work Group, Highlights and Executive Summary. Bethesda, National Institutes of Health, 2012.

6 United Nations Office on Drugs and Crime. World Drug Report 2012. Vienna, United Nations, 2012.

7 United Nations Office on Drugs and Crime. World Drug Report 2013. Vienna, United Nations, 2013.

8 Volkow ND, Fowler JS, Wang GJ, et al. Distribution and pharmacokinetics of methamphetamine in the human body: clinical implications. PLoS ONE 2010; 5: e15269.

9 Won S, Hong RA, Shohet RV, et al. Methamphetamine-associated cardiomyopathy. Clin Cardiol 2013; 36: $737-742$.

10 Westover AN, Nakonezny PA. Aortic dissection in young adults who abuse amphetamines. Am Heart J 2010; 160 315-321.

11 Zamanian RT, Hedlin H, Greuenwald P, et al. Features and outcomes of methamphetamine associated pulmonary arterial hypertension. Am J Respir Crit Care Med 2017; 196: e32-e47.

12 Megarbane B, Chevillard L. The large spectrum of pulmonary complications following illicit drug use: features and mechanisms. Chem Biol Interact 2013; 206: 444-451.

13 Gruenewald PJ, Ponicki WR, Remer LG, et al. Mapping the spread of methamphetamine abuse in California from 1995 to 2008. Am J Public Health 2013; 103: 1262-1270.

14 Wiley LK, Shah A, Xu H, et al. ICD-9 tobacco use codes are effective identifiers of smoking status. J Am Med Inform Assoc 2013; 20: 652-658.

15 Gruenewald PJ, Johnson FW, Ponicki WR, et al. Assessing correlates of the growth and extent of methamphetamine abuse and dependence in California. Subst Use Misuse 2010; 45: 1948-1970.

16 Almeida RR, Zanetti G, Souza AS Jr, et al. Cocaine-induced pulmonary changes: HRCT findings. J Bras Pneumol 2015; 41: 323-330. 
17 O'Donnell AE, Mappin FG, Sebo TJ, et al. Interstitial pneumonitis associated with "crack" cocaine abuse. Chest 1991; 100: 1155-1157.

18 Wilson KC, Saukkonen JJ. Acute respiratory failure from abused substances. J Intensive Care Med 2004; 19: 183-193.

19 van der Klooster JM, Grootendorst AF. Severe bullous emphysema associated with cocaine smoking. Thorax 2001; 56: 982-983.

20 Rome LA, Lippmann ML, Dalsey WC, et al. Prevalence of cocaine use and its impact on asthma exacerbation in an urban population. Chest 2000; 117: 1324-1329.

21 Zein JG, Erzurum SC. Asthma is different in women. Curr Allergy Asthma Rep 2015; 15: 28.

22 Celli B. Sex differences in mortality and clinical expressions of patients with chronic obstructive pulmonary disease. Am J Respir Crit Care Med 2011; 183: 317-322.

23 Falagas ME, Mourtzoukou EG, Vardakas KZ. Sex differences in the incidence and severity of respiratory tract infections. Respir Med 2007; 101: 1845-1863.

24 R Core Team. R: A Language and Environment for Statistical Computing. Vienna, R Foundation for Statistical Computing, 2017. www.R-project.org/

25 Venables W, Ripley B. Modern Applied Statistics with S. 4th Edn. New York, Springer, 2002.

26 Haim DY, Lippmann ML, Goldberg SK, et al. The pulmonary complications of crack cocaine. Chest 1995; 107: $233-240$

27 Tashkin D. Effects of marijuana smoking on the lung. Ann Am Thorac Soc 2013; 10: 239-247.

28 Salamanca SA, Sorrentino EE, Nosanchuk JD, et al. Impact of methamphetamine on infection and immunity. Front Neurosci 2015; 8: 1-12.

29 Martinez LR, Mihu MR, Gácser A, et al. Methamphetamine enhances histoplasmosis by immunosuppression of the host. J Infect Dis 2009; 200: 131-141.

30 Nestor TA, Tamamoto WI, Kam TH, et al. Acute pulmonary oedema caused by crystalline methamphetamine. Lancet 1989; 334:1277-1278.

31 Chen P, Cao A, Miyagawa $\mathrm{K}$, et al. Amphetamines promote mitochondrial dysfunction and DNA damage in pulmonary hypertension. JCI Insight 2017; 2: e90427.

32 Downey GP, Dong Q, Kruger J, et al. Regulation of neutrophil activation in acute lung injury. Chest 1999; 116: 46S-54S.

33 Wells SM, Buford MC, Braseth SN, et al. Acute inhalation exposure to vaporized methamphetamine causes lung injury in mice. Inhal Toxicol 2008; 20: 829-838.

34 Strategic Intelligence Section. 2017 National Drug Threat Assessment. Springfield, US Dept of Justice Drug Enforcement Administration, 2017. 